\title{
RESPONSE SURFACE METHODOLOGY AND ITS APPLICATION IN SIMULATION
}

\author{
Sarah J. Hood \\ Peter D. Welch \\ IBM T.J. Watson Research Center \\ Yorktown Heights, NY 10598
}

\begin{abstract}
The contents of this paper reflect a portion of the conference tutorial on the application of response surface methodology to simulation. In this paper we will present an outline of the methodology and give a simple example of its application to a two variable optimization problem. In the tutorial itself we will discuss the methodology in greater detail and discuss an additional application to a six variable optimization problem. The examples are taken from the simulation of a semiconductor manufacturing line.
\end{abstract}

\section{INTRODUCTION}

This is a discussion of the application of the statistical methodology of response surface exploration to simulation. Specifically, it is a discussion of the methodology summarized in the book "Empirical Model Building and Response Surfaces" by Box and Draper (1987).

We assume that the simulation model has $K$ continuous parameters $\theta_{1}, \ldots, \theta_{K}$ and that we are interested in a performance characteristic, $C\left(\theta_{1}, \ldots, \theta_{K}\right)$, of the model, which is the expected value of an output random variable, $Y\left(\theta_{1}, \ldots, \theta_{K}\right)$. In the examples we will consider, $C$ is a cost function derived from the expected cycle time in a model of a semiconductor manufacturing line. The cycle time is the time from beginning of manufacture to completion. We are interested in "exploring" the surface $C\left(\theta_{1}, \ldots, \theta_{K}\right)$. $Y\left(\theta_{1}, \ldots, \theta_{K}\right)$ will be the sample mean of the cost function over a simulation run.

The methodology we will describe involves the "classical" application of experimental design based on standard least squares theory. We assume that $C\left(\theta_{1}, \ldots, \theta_{K}\right)$ is smooth enough so that it can be approximated by either a first or second degree polynomial over the sequence of regions of exper- imental activity. The methodology is sequential in nature with each successive experiment building on the results and insights of earlier experiments. Thus, it is ideally suited to simulation because of the relative ease with which data can be obtained in the simulation context.

In section 2 we will discuss the fitting of first order polynomials. In section 3 we will discuss the fitting of second order polynomials (quadratics). In section 4 we will combine the results of sections 2 and 3 and discuss the optimization problem; i.e., the location of maxima or minima. We will give a simple example illustrating the technique on a semiconductor manufacturing model with two variables. The talk will go into much greater detail than the paper in all aspects of the methodology. Further, in the talk, in addition to the two variable example, we will give a six variable example.

\section{FIRST ORDER MODELS}

In fitting a first order model we assume that, in the region over which we conduct the experiment, $C\left(\theta_{1}, \ldots, \theta_{K}\right)$ can be approximated by a function of the form

$$
C\left(\theta_{1}, \ldots, \theta_{K}\right)=a_{0}+\sum_{k=1}^{K} a_{k} \theta_{k}
$$

i.e. by a first order polynomial. To explore this hypothesis the methodology uses 2 level fractional factorial designs with center points. In these designs a rectangular region of the $\left(\theta_{1}, \ldots, \theta_{K}\right)$ space is defined by specifying two levels for each of the parameters, i.e. by specifying $\theta_{k 1}<\theta_{k 2} k=1, \ldots, K$. These sets of levels would be such that, in the context of the application, it would make sense to explore a first order model over the region so defined. In the application of 2-level fractional factorial designs the 
$\left(\theta_{1}, \ldots, \theta_{R}\right)$ coordinate system is replaced with the coordinates $\left(x_{1}, \ldots, x_{K}\right)$ where

$$
x_{k}=\frac{\theta_{k}-\left(\theta_{k 1}+\theta_{k 2}\right) / 2}{\left(\theta_{k 2}-\theta_{k 1}\right) / 2}
$$

The $x_{k}$ coordinates take on the value -1 for the lower level, $\theta_{k 1}$, and +1 for the upper level, $\theta_{k 2}$. Equation 1 then becomes

$$
C\left(x_{1}, \ldots, x_{K}\right)=\mu+\sum_{k=1}^{K} \alpha_{k} x_{k}
$$

$C\left(\theta_{1}, \ldots, \theta_{K}\right)$ can be obtained by substituting (2) into (3). The designs assume that the output random variable $Y\left(x_{1}, \ldots, x_{K}\right)$ has expectation $C\left(x_{1}, \ldots, x_{R}\right)$ plus independent, identically distributed, Gaussian noise. In the simulation environment the assumptions of independence and normality do not usually present a problem but the assumption that the noise has a variance independent of $\left(x_{1}, \ldots, x_{K}\right)$ is sometimes troublesome. For further discussion of this issue, see Welch (1990).

Two level fractional factorial designs possess a number of desirable features. First they provide a hierarchy of designs for fitting a hierarchy of more and more complicated two level models. The model of equation (3) is the simplest. This model assumes that each factor has an additive effect which is independent of the level settings of the other factors. If this model is true the methodology of two level fractional factorial designs provides resolution 3 designs which enable you efficiently obtain unbiased estimates of the parameters $\mu$ and $\alpha_{1}, \ldots, \alpha_{K}$.

The next most complicated model assumes that

$$
C\left(x_{1}, \ldots, x_{K}\right)=\mu+\sum \alpha_{k} x_{k}+\sum_{i<j} \beta_{i j} x_{i} x_{j}
$$

It is again additive. It assumes that the effect of changing the level of a parameter depends on the values of the other parameters but only in a pair wise fashion and in an additive way. If this more complicated model is true the methodology provides resolution 4 designs which generate unbiased estimates of the $\alpha_{k}$ independent of the values of the $\beta_{i j}$ and resolution 5 designs which provide unbiased estimates of all the $\alpha_{k}$ and $\beta_{i j}$. The full quadratic model which we will consider in the next section is

$$
C\left(x_{i}, \ldots, x_{K}\right)=\mu+\sum \alpha_{k} x_{k}+\sum_{i \leq j} \beta_{i j} x_{i} x_{j} .
$$

We are interested in the first order model because it may constitute a sufficiently accurate model over the region of immediate investigation. Further in the case of all three models the coefficients $\alpha_{1}, \ldots, \alpha_{k}$ define the path of steepest descent (ascent) which we shall see is critical to the optimization problem we will consider in the examples. In our examples, we will be selecting a minimum cost system. Hence, we will be interested in the path of steepest descent. If we let $L=\left(\sum \alpha_{k}^{2}\right)^{1 / 2}$ then a vector of unit length in this direction has coordinates $-\alpha_{1} / L, \ldots,-\alpha_{K} / L$.

In applying this methodology to these optimization problems we proceed as we will now outline. An initial experimental region is selected. A first order model is fit, checked to see if it is a reasonable fit and checked to make sure that a minimum does not fall within the region. An outline of the process for accomplishing this will be given below. If it is decided that the first order model is valid, the path of steepest descent is followed to an estimated minimum of the cost function along this path. This procedure is then repeated for a region about this minimum point. If it is determined that it is likely that a minimum exists within the region then, as will be outlined in the next section, the design is augmented and a second order model is fit. This model is analyzed to determine whether or not a minimum exists and so on.

To begin the process an initial design region is chosen by selecting the levels $\theta_{k 1}<\theta_{k 2} k=1, \ldots, K$. Ideally, a resolution 5 design is run with center points. Center points are replications at the center of the region, i.e. at the point $x_{1}=\cdots=x_{K}=0$. Then the standard techniques of 2 level fractional factorial model analysis are applied. These include confidence intervals on the coefficients, probability plots, residual plots, etc. The center points provide a curvature check. In particular

$$
\begin{aligned}
& c=\text { average response over 2-level factorial } \\
& \text { runs - average response over center points }
\end{aligned}
$$

provides an estimate of $\sum \beta_{k k}$. If there is a minimum the $B_{k k}$ are all positive and hence the magnitude of $c$ should be compared to the magnitude of the estimated coefficients. If $c$ is large with respect to the $\alpha_{k}$, a full quadratic model should be fit.

In this process confidence intervals can be placed on the direction of steepest descent as well as on the 
coefficients and the curvature. If any of these estimates are not deemed accurate enough, additional replications and/or center points can be added to the design and the analysis repeated.

There are two elements in the design of experiments which are important in other applications but would usually not be relevant in simulation applications: blocking and randomization. They are designed to control the effects of variations in experimental conditions by either effectively including these variations in the model or by randomizing the order of the sequence of experimental trials. Such variations would not appear to occur in the more pure world of simulation.

\section{SECOND ORDER MODELS}

Second order models cannot be fit with 2 level designs plus center points. Hence if, as the sequence of experiments is proceeding, the $\beta_{i j}$ are not small with respect to the $\alpha_{k}$ and/or the estimate of curvature is high, a second order model will have to be fit. In fitting a second order model there is a class of central composite designs which is appealing for two reasons. First, they can be obtained by augmenting resolution 5 , fractional factorial designs. Hence, they fit well into the sequential development of an investigation. Second, they can be constructed so as to be rotatable; a concept we will discuss next.

To standardize the variance of our estimate of the theoretical surface we generate a function

$$
V\left(x_{1}, \ldots, x_{K}\right)=\left(n / \sigma^{2}\right) \operatorname{Variance}\left(\hat{C}\left(x_{1}, \ldots, x_{K}\right)\right)
$$

called the variance function. Here $\mathrm{n}$ is the total number of runs and $\sigma$ is the standard deviation of the outcome of each run. The information function is defined as

$$
I\left(x_{1}, \ldots, x_{K}\right)=V^{-1}\left(x_{1}, \ldots, x_{K}\right)
$$

Now a design is rotatable if $V$ and hence $I$ are a function of $x_{1}, \ldots, x_{K}$ only through

$$
\rho=\left(x_{1}^{2}+\cdots+x_{K}^{2}\right)^{1 / 2}
$$

That is, if $V$ and hence $I$ are only a function of the distance from the origin in $x$ space. Rotatability is a desirable feature since a priori there is usually no reason to favor any particular direction. $I\left(x_{1}, \ldots, x_{K}\right)$ will, in general, go to zero as the distance of the point $x_{1}, \ldots, x_{K}$ moves away from the origin.

Central composite designs are obtained from resolution 5 , fractional factorial designs by the addition of star points and perhaps more center points. Star points are points where one of the $x_{i}$ takes on the values $\pm \alpha$ while the remaining $x_{i}$ are all 0 . For example the pair of star points for the first variable would be $(\alpha, 0, \ldots, 0)$ and $(-\alpha, 0, \ldots, 0)$. Thus, for a design of $K$ variables there would be $2 K$ star points. When augmenting the 2 level design, the $2 K$ star points can be replicated any number of times and additional center points added. Using the notation of Box and Draper the $\alpha$ that generates a rotatable design is given by

$$
\alpha=\left(\frac{\left(2^{k-p}\right) r_{c}}{r_{s}}\right)^{1 / 4}
$$

where $k$ is the number of factors, a $2^{k-p}$ fractional factorial design of resolution 5 is being augmented, $r_{c}$ is the number of replications of the fractional factorial design and $r_{s}$ is the number of replications of the $2 K$ star points. The above function for $\alpha$ is equation (14.3.14) of Box and Draper. The information function of the design is given as a function of $\rho$ by equations (14.3.13) and (14.3.15) of Box and Draper. The information function is a function of $2^{k-p}, r_{c}, r_{s}$ and the total number of center points.

Once the central composite design has been generated, the runs are made and the results are analyzed. The coefficients are generated, confidence intervals produced, and the model checked using standard least squares methodology. The fitted second order model will have a stationary point. The experimenter will be interested in the characteristics of this stationary point and its location relative to the region of the design. There are two canonical forms of the model which are useful for interpreting the stationary point and the behavior of the fitted surface in the region of the design. In the type $A$ canonical form the axes are rotated so as to remove the cross product terms. Thus we have new variables $y_{1}, \ldots, y_{K}$ and a fitted function of the form

$$
\hat{C}\left(y_{1}, \ldots, y_{K}\right)=a_{0}+\sum a_{i} y_{i}+\sum b_{i i} y_{i}^{2}
$$

This is useful for interpreting the behavior of the response surface when the stationary point is some distance from the experimental region. For a dis- 
cussion of this case see Chapters 10 and 11 of Box and Draper. In the type B canonical form, the origin of the coordinate system is translated to stationary point and hence, we have coordinates $z_{1}, \ldots, z_{K}$ and

$$
\hat{C}\left(z_{1}, \ldots, z_{K}\right)=d_{0}+\sum b_{i i} z_{i}^{2}
$$

This is useful for interpreting the behavior of the response surface when the stationary point is within the experimental region. In both canonical forms the signs of the $b_{i i}$ determine the type of stationary point. If it is a minimum, which is what we will be looking for in our examples, the $b_{i i}$ must all be positive.

\section{A SIMPLE OPTIMIZATION EXAMPLE IN TWO DIMENSIONS}

\subsection{The Model}

We will consider the model of semiconductor manufacturing line logistics which was discussed in Hood, Amamoto and Vandenberge (1989) and Hood and Welch (1992). In this model, the line is subject to interruptions for tool set up, preventative maintenance and repair of failures. These interrupt processes are modeled as the highest priority sources of work for the tool groups. The interruptions (arrivals) and interrupt durations (service times) have i.i.d. exponential distributions. Hence, there are just two parameters for each interrupt process: the mean time between interrupts and the mean interrupt duration. In this simple example, we will consider only the setup interrupt process which was the process having the strongest effect on model behavior. Hence, we will be considering two model parameters

$$
\begin{aligned}
& \theta_{1}=\text { mean time between setup interrupts } \\
& \theta_{2}=\text { mean duration of setup interrupts. }
\end{aligned}
$$

In the talk we will, in addition, consider a six parameter example involving all three processes. The response variable, $C$ is a function of $R$, the steady state mean cycle time: the mean elapsed time from entry into the manufacturing line until completion.

In the application of the model there is a base case, a reference case which has model parameters judged to best approximate a planned line. We will be interested in optimizing over a region representing possible improvements on this base case. The reference mean values (corresponding to the base case) for $\theta_{1}$ and $\theta_{2}$ are taken to be unity. An increase in $\theta_{1}$ or a decrease in $\theta_{2}$ will reduce the mean cycle time which is beneficial. The range of $\theta_{1}, \theta_{2}$ we will consider is $1 \leq \theta_{1} \leq 2$ and $.5 \leq \theta_{2} \leq 1$. The function $R\left(\theta_{1}, \theta_{2}\right)$ was estimated in extensive simulation and the estimated function is plotted in figure 1 .

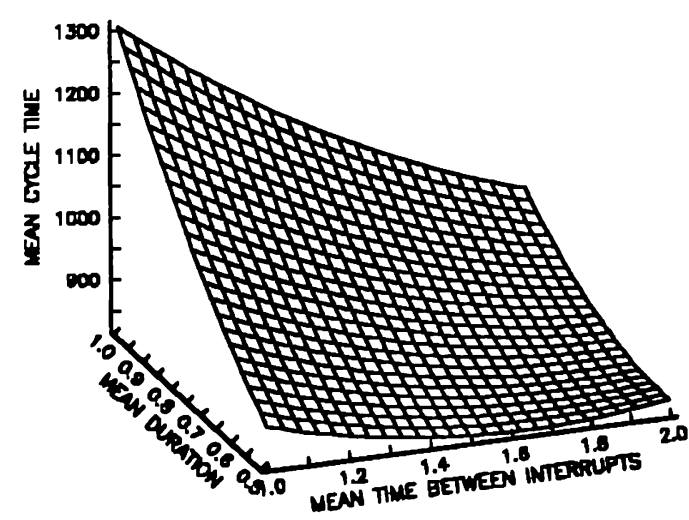

Figure 1. Steady State Mean Cycle Time

Now to illustrate the application of the methodology, we need a cost function which has a minimum value. We generated such a function by assuming the cost to be given by

$$
C\left(\theta_{1}, \theta_{2}\right)=R\left(\theta_{1}, \theta_{2}\right)+200\left(\theta_{1}-1\right)^{2}+1200\left(1-\theta_{2}\right)^{2}
$$

This cost function is plotted in figure 2. This function was developed to provide an example for the methodology. We wanted a function which possessed a minimum in a region of feasible values for the parameters. Its form is reasonable, with $R\left(\theta_{1}, \theta_{2}\right)$ representing the inventory cost of the work in progress and the other two terms representing the costs associated with realizing improvements in $\theta_{1}$ and $\theta_{2}$.

Hence each time we make a simulation run we generate a random variable $Y\left(\theta_{1}, \theta_{2}\right)$ given by

$$
Y\left(\theta_{1}, \theta_{2}\right)=C\left(\theta_{1}, \theta_{2}\right)+\epsilon
$$

where $\epsilon$ is an error random variable. We are interested making a series of simulation runs as the elements of a sequence of experimental designs to estimate the location of the minimum point of $C\left(\theta_{1}, \theta_{2}\right)$. That is, we are interested in pursuing the methodology described in Sections 2 and 3. 


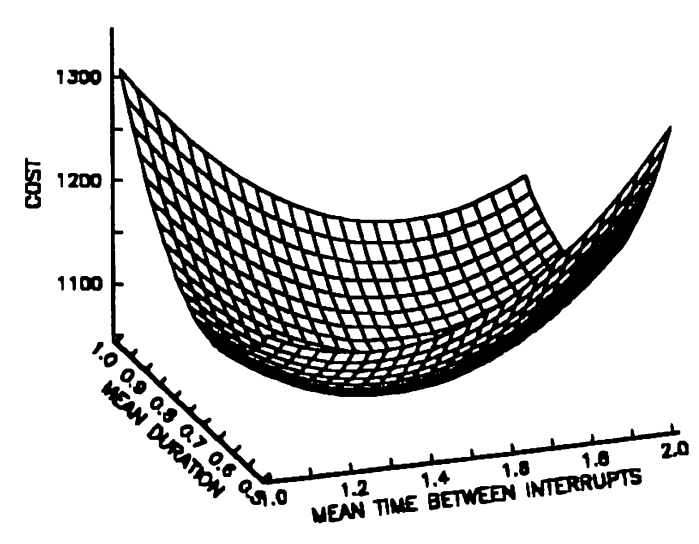

Figure 2. Cost Function

In creating this example, we did not actually run the simulation model, but rather simulated its running. Since we knew the function $C\left(\theta_{1}, \theta_{2}\right)$, we could simulate the random variables $Y\left(\theta_{1}, \theta_{2}\right)$ by adding the noise random variable. In the earlier set of simulation runs that estimated $R\left(\theta_{1}, \theta_{2}\right)$, it was determined that for a run of reasonable length the noise random variables were normally distributed with a standard deviation of 20 . In the talk we will give an example with six variables where the simulation model was run to produce each data point. This two variable problem is designed to illustrate the essential features of the methodology in an environment where the important features of its operation can be easily visualized.

\subsection{The Experiment}

In the work to be described next, we used an experimental extension of the IBM package "A Graphical Statistical System (AGSS)". (See Lane and Welch (1987).) This package contains software for two level fractional factorial experiments with center points. For the purposes of this study we worked with an experimental internal IBM version which also includes central composite designs.

We started the process of finding the minimum of $C\left(\theta_{1}, \theta_{2}\right)$ in a region in the immediate vicinity of the base case. We ran a 2 level experiment (with center points) with the levels of $\theta_{1}$ being 1 and 1.2 and the levels of $\theta_{2}$ being .9 and 1 . We ran a full factorial design replicated 5 times with 5 center points. The resulting coefficient confidence interval table is shown in table 1. Notice that the main effects are large with respect to the interaction effect and that the main effects are statistically significant whereas the interaction effect is not. Further, notice that the curvature is small and statistically insignificant. Hence, a first order fit is reasonable and we proceed to calculate and explore the path of steepest descent.

However, at this point it is important to emphasize the relationship of these kinds of results to the ease of data acquisition and the consequent potential flexibility in simulation experimentation. We conducted a single experiment with five replications and then viewed the results. If they had been less convincing, we could have added more replications and viewed the results of the extended experiment continuing this process until we became convinced either to move along the estimated path of steepest descent or to fit a second order model. As alternatives, we could have viewed the results after each replication or implemented a decision procedure in a higher level program which automatically took more data, made the requisite decisions and searched for a minimum. Further such an automatic procedure could keep track of all its steps so its "reasoning" could be reviewed post facto by the experimenter. For further discussion of the potential of such higher level systems, see MacNair and Welch (1991).

We return now to our search for the minimum of $C\left(\theta_{1}, \theta_{2}\right)$. From the estimated coefficients of table 1 we have that the estimate of the vector of unit length in the direction of steepest descent is given by

$$
(.6894,-7244)=(77.207,-81.118) / \mathrm{L}
$$

where $L=\left(77.207^{2}+81.118^{2}\right)^{5}$. This direction, however, is relative to the coordinate system $x_{1}, x_{2}$ of the first design, not the coordinate system $\theta_{1}, \theta_{2}$. Hence, we have to make the appropriate transformation to generate a sequence of points along the direction of steepest descent starting from the center of the initial design, the point $\left(\theta_{1}, \theta_{2}\right)=(1.1, .95)$. We explored $C\left(\theta_{1}, \theta_{2}\right)$ along this path to estimate the point at which it assumes a minimum. This estimated minimum point will be the center of the second design.

In estimating this minimum point we estimated $C\left(\theta_{1}, \theta_{2}\right)$ at a sequence of eight equally spaced points each separated by a unit distance in $\left(x_{1}, x_{2}\right)$ coordinates. At each point we made 16 replications (i.e. 16 simulated runs). The estimates of $C\left(\theta_{1}, \theta_{2}\right)$ along with $95 \%$ confidence intervals are shown in figure 3. Notice there is a shallow minimum at the fifth point. We selected the location of this fifth point as the center for the second design. It is located at the 


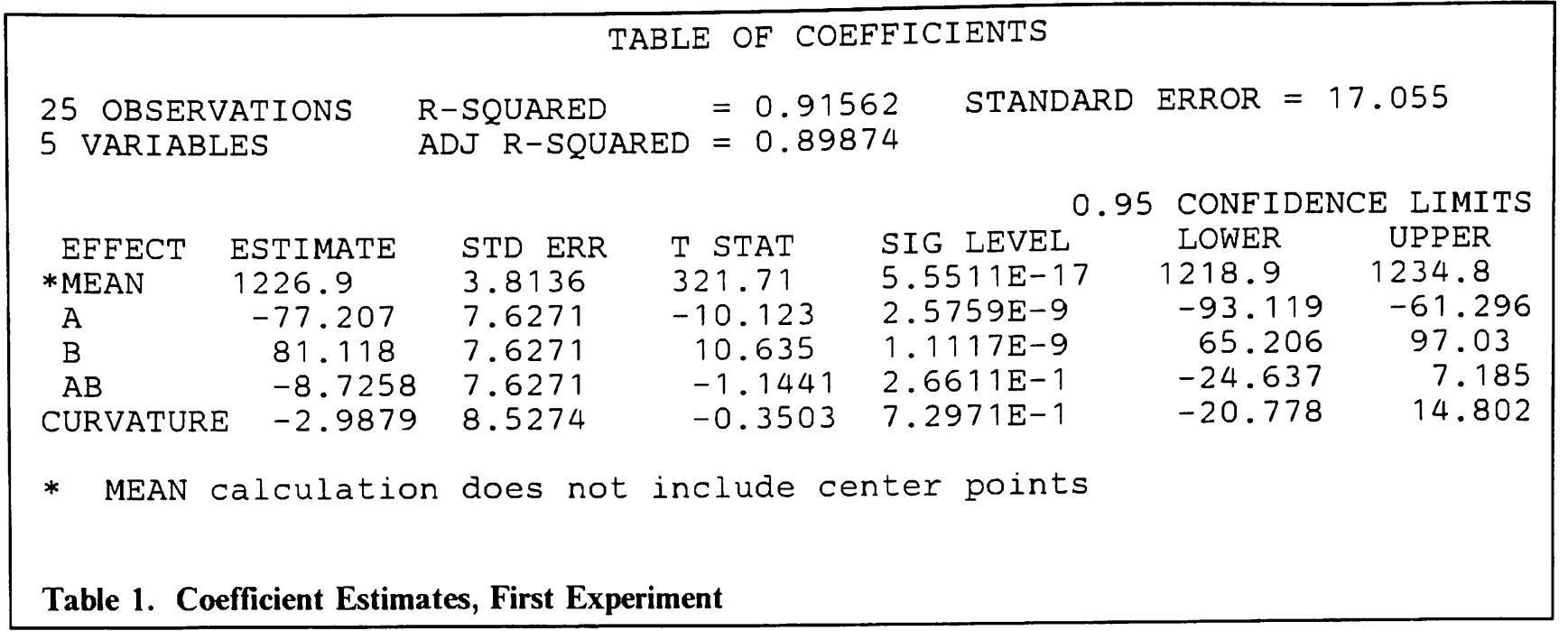

point $\left(\theta_{1}, \theta_{2}\right)=(1.4434, .76825)$. Again this specific process could be done sequentially in a number of ways and could be automated.

With this point as center and with the same spacing we repeated the initial experiment. That is we ran a 2 factor, full factorial experiment with the $\theta_{1}$ levels at 1.3434 and 1.5434 and the $\theta_{2}$ levels at .71825 and .81825 . Again, we replicated the design 5 times and added 5 center points, exactly as in the initial experiment. The only change was in the location of the center point.

The resulting table of coefficient estimates is shown in table 2 . Here, notice the two way interaction term is of the same rough magnitude as the main effects. All are relatively small as is the curvature estimate indicating the possible presence of a shallow minimum.

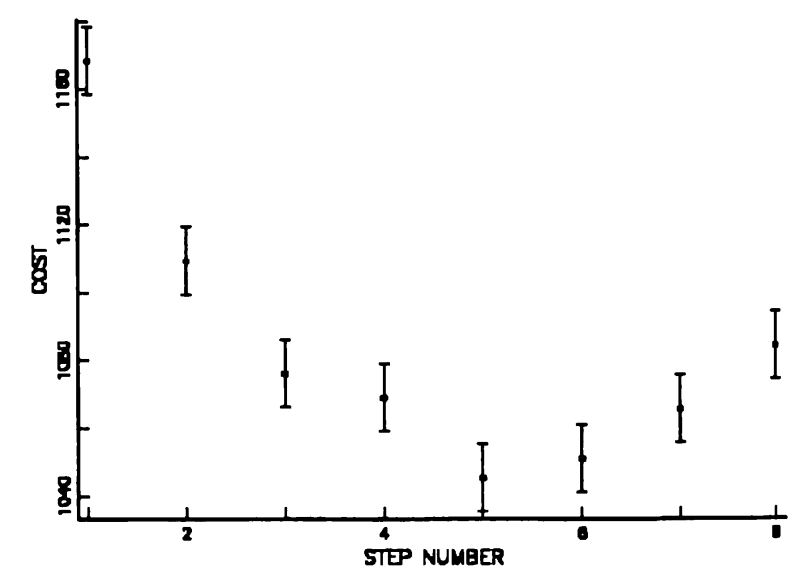

Figure 3. Estimate of Cost Function Along Path of Steepest Descent

TABLE OF COEFFICIENTS

$\begin{array}{lll}25 \text { OBSERVATIONS } & \text { R-SQUARED } & =0.09346\end{array} \quad$ STANDARD ERROR $=17.364$

\begin{tabular}{|c|c|c|c|c|c|c|}
\hline \multicolumn{7}{|r|}{6} \\
\hline EFFECT & ESTIMATE & STD ERR & T STAT & SIG LEVEL & LOWER & UPPER \\
\hline *MEAN & 1054.6 & 3.8827 & 271.62 & $8.3267 E-17$ & 1046.5 & 1062.7 \\
\hline A & -4.122 & 7.7655 & -0.53081 & $6.0140 E-1$ & -20.322 & 12.078 \\
\hline B & 4.1987 & 7.7655 & 0.54069 & $5.9469 E-1$ & -12.002 & 20.399 \\
\hline $\mathrm{AB}$ & -8.463 & 7.7655 & -1.0898 & $2.8875 \mathrm{E}-1$ & -24.663 & 737 \\
\hline CURVATU & -4.7562 & 8.6821 & -0.54782 & $5.8988 E-1$ & -22.869 & 13.356 \\
\hline
\end{tabular}

* mean calculation does not include center points

Table 2. Coefficient Estimates, Second Experiment 
Hence this second experiment was augmented to create a rotatable central composite design capable of estimating the full second order model. The star portion was replicated 5 times so, from equation 6 , $\alpha=2^{s}=1.414$. In figure 4 we show the information function with 5 additional center points added. Notice the decrease in information near the center of the design. To remedy this we added 20 center points. The resulting information function is plotted in figure 5. This is the information function for the experiment performed.

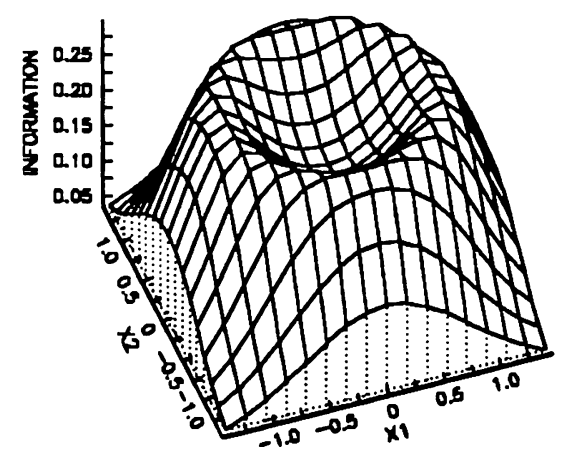

Figure 4. Information Function for Central Composite Design with 5 Additional Center Points

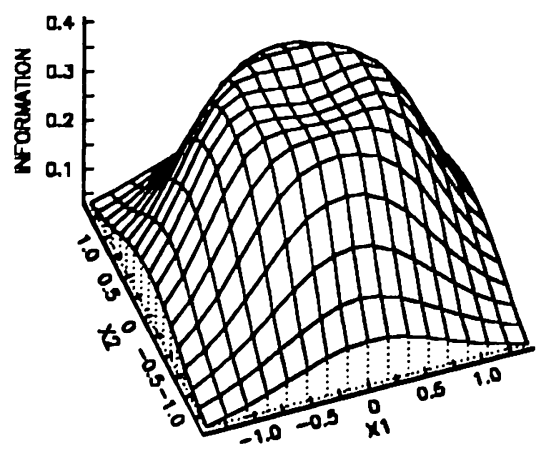

Figure 5. Information Function for Central Composite Design with 20 Additional Center Points

The analysis of this central composite design indicated a minimum within the experimental region.
The table giving this information is shown in table 3. Notice from the signs of the squared terms in the $\mathrm{A}$ and $\mathrm{B}$ canonical forms that the stationary point is a minimum. The location of the stationary point is given in terms of the $x_{1}, x_{2}$, coordinates relative to the new center. The estimated location of the minimum in $\theta_{1}, \theta_{2}$ coordinates is $1.4457, .7415$. The estimated minimum value is 1046.1. In Figure 6 we have plotted the contour function of the fitted quadratic over the experimental region. The best way to view the behavior of the fit in this case is in terms of the type B canonical form. The contours of constant cost are ellipses centered at the minimum point.

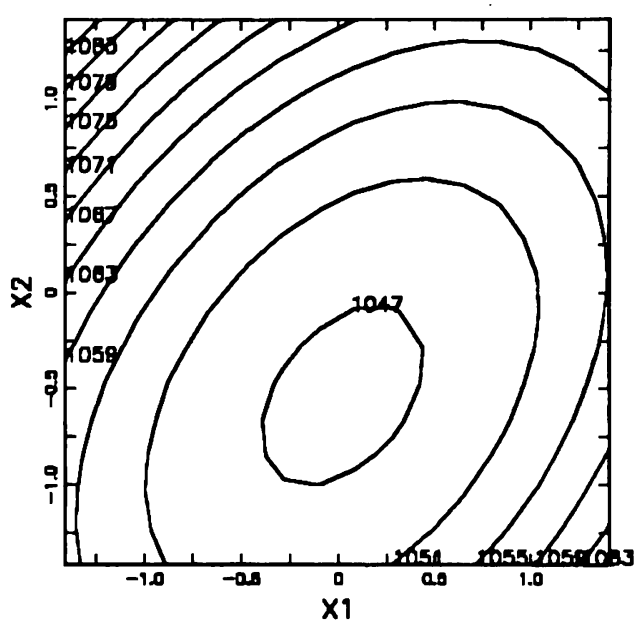

Figure 6. Contour Plot of Fitted Quadratic Over the Final Experimental Region

\section{SUMMARY}

This has been a brief discussion on the application of the response surface exploration methodology of Box and Draper (1987) to simulation. This methodology has a number of features which fit well into the framework of simulation experimentation. It is sequential so it matches well the ready availability of data in the simulation context. It is sequential both in the model fitting and estimation at each experimental stage and in the generation of a sequence of experimental stages. It produces confidence intervals on the estimates of interest and powerful diagnostics for the model fitting. It provides a solid theoretical and intuitive base in classical regression theory for the experimental process so that people applying it should know at all times where they are and what they are about. 


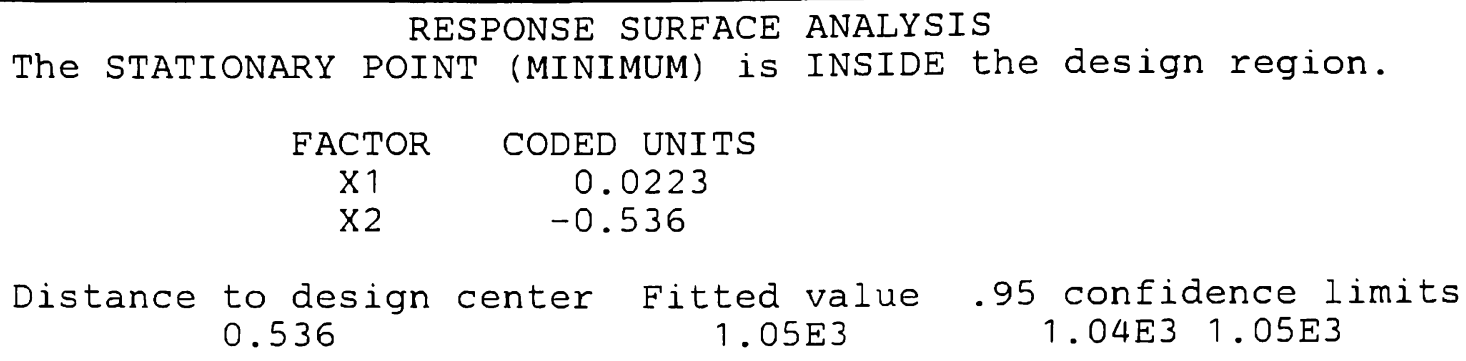

THE A CANONICAL FORM:

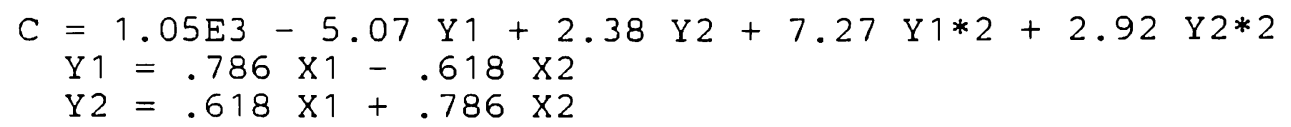

THE B CANONICAL FORM:

$$
\begin{gathered}
C=1.05 \mathrm{E} 3+7.27 \mathrm{Z} 1 * 2+2.92 \mathrm{Z} 2 * 2 \\
\mathrm{Z} 1=.786 \times 1-.618 \times 2-.348 \\
\mathrm{Z} 2=.618 \times 1+.786 \times 2+.408
\end{gathered}
$$

Table 3. Response Surface Analysis: Third Experiment

\section{REFERENCES}

Box, G.E.P., and Draper, N.R. 1987. Empirical model-building and response surfaces, New York: John Wiley \& Sons.

Hood S.J., A.E. Amamoto, and A.T. Vandenberge. 1989. A modular structure for a highly detailed model of semiconductor manufacturing. Proceedings of the 1989 Winter Simulation Conference. E.A. MacNair, K.J. Musselman, and P. Heidelberger, Eds. IEEE, Piscataway, NJ, 811-817.

Hood S.J. and Welch, P.D. 1992. Experimental design issues in simulation with examples from semiconductor manufacturing. Proceedings of the 1992 Winter Simulation Conference. J.J. Swain, D. Goldsman, R.C. Crain, and J.R. Wilson, Eds. IEEE, Piscataway, NJ, 255-263.

Lane, T. and Welch, P.D. 1987. The integration of a menu-oriented graphical statistical system with its underlying general purpose language. Computer Science and Statistics: Proceedings of the 19th Symposium of the Interface. American Statistical Association, 267-273.

MacNair, E.A. and Welch, P.D. 1991. Toward a higher level, output analysis interface. Proceedings of the 1991 Winter Simulation
Conference. B.L. Nelson, W.D. Kelton, and G.M. Clark, Eds. IEEE, Piscataway, NJ, 822-831. Welch, P.D. 1990. Simulation and regression: dealing with the assumption of a common error variance. Proceedings of the 1990 Winter Simulation Conference. O. Balci, R.P. Sadowski, and R.E. Nance, Eds. IEEE, Piscataway, NJ, 392-394.

\section{AUTHOR BIOGRAPHIES}

SARAH J. HOOD is a Research Staff Member in the Manufacturing Research Department of the IBM Thomas J. Watson Research Center in Yorktown Heights NY. She received a Ph.D. in Mechanical Engineering from the U. of California, Davis. She is currently working in the discrete event system domain exploring various methods and tools for decision support of semiconductor manufacturing lines. She has also simulated environmental, physiological, and electro-mechanical systems. She is a member of SCS and ORSA.

PETER D. WELCH is a Research Staff Member in the Computer Science Department at the IBM Thomas J. Watson Research Center in Yorktown Heights NY. He received a Ph.D. in Mathematical Statistics from Columbia University. His research interests are graphical-statistical software and simulation output analysis. He is a member of ORSA. 